\title{
Ruptura de saco herniario umbilical con evisceración por colocación de cuerpo extraño. Reporte de caso
}

\section{Rupture of umbilical hernia sack with evisceration by placing foreign body. Case report}

\author{
Laura E González-Rodríguez, ${ }^{1}$ Oliver A Toledo-Sánchez, ${ }^{2}$ Elizabeth Islas-Sánchez ${ }^{2}$
}

\begin{abstract}
Resumen
ANTECEDENTES: La herniorrafia umbilical es una de las intervenciones quirúrgicas que más se realizan en pacientes pediátricos; su tratamiento ha cambiado poco en los últimos 100 años. Con frecuencia se recomendaba la compresión externa e insistía en la importancia de la preservación de la apariencia del ombligo, en lugar de la escisión. Las complicaciones son excepcionales, por lo que la ruptura espontánea de la hernia umbilical es extremadamente rara.

CASO CLíNICO: Lactante femenina de dos meses, previamente sana, con ruptura de un saco herniario umbilical asociado con la colocación de un cuerpo extraño, con evisceración del intestino delgado. El cuadro clínico fue de corta evolución pues la reparación quirúrgica fue inmediata, con recuperación posoperatoria sin contratiempos; requirió breve tratamiento intrahospitalario.

CONCLUSIONES: Si bien las indicaciones para la reparación quirúrgica de la hernia umbilical se han debatido y pueden variar entre los cirujanos pediatras, el riesgo de complicaciones es muy bajo; los casos de mortalidad reportados son pocos. Los médicos y los padres deben estar informados y ser capaces de identificar los signos de advertencia de ruptura espontánea.
\end{abstract}

PALABRAS CLAVE: Herniorrafia; pacientes pediátricos; ruptura espontánea; hernia umbilical; evisceración; intestino delgado.

Abstract

BACKGROUND: Umbilical herniorrhaphy is one of the most performed surgical interventions in pediatric patients; his treatment has changed little in the last 100 years. External compression was often recommended and insisted on the importance of preserving the appearance of the navel, rather than excision. The complications are exceptional, so the spontaneous rupture of the umbilical hernia is extremely rare.

CLINICAL CASE: Female infant of two months, previously healthy, with rupture of an umbilical hernial sac associated with the placement of a foreign body, with evisceration of the small intestine. The clinical picture was of short evolution because the surgical repair was immediate, with postoperative recovery without setbacks; It required brief intrahospital treatment.

CONCLUSIONS: Although the indications for surgical repair of umbilical hernia have been debated and may vary among pediatric surgeons, the risk of complications is very low; The cases of mortality reported are few. Doctors and parents should be informed and be able to identify warning signs of spontaneous rupture.

KEYWORDS: Herniorrhaphy; Pediatric patients; Spontaneous rupture; Umbilical hernia; Small intestine.

\begin{abstract}
${ }^{1}$ Médico pediatra, residente de Cirugía pediátrica.

${ }^{2}$ Médico pediatra y cirujano pediatra. Hospital Pediátrico Moctezuma, Secretaría de Salud del Gobierno de la Ciudad de México.
\end{abstract}

Recibido: 20 de abril 2018

Aceptado: 4 de diciembre 2018

Correspondencia

Laura Edith González Rodríguez

auraled_61@hotmail.com

Este artículo debe citarse como González-Rodríguez LE, Toledo-Sánchez OA, Islas-Sánchez E. Ruptura de saco herniario umbilical con evisceración por colocación de cuerpo extraño. Reporte de caso. Acta Pediatr Mex. 2019;40(2):65-70. 


\section{ANTECEDENTES}

Las hernias umbilicales en niños ocurren con la misma frecuencia en uno y otro sexo. Casi todas se reconocen después de la separación del cordón umbilical en las primeras semanas de vida y la mayor parte se observan a los seis meses de edad. Muchas hernias cierran espontáneamente durante los primeros tres años de vida. ${ }^{1,2}$

El tamaño del defecto de la hernia es uno de los principales factores determinantes del cierre espontáneo, la mayor parte mide menos de 0.5 $\mathrm{cm}$ y cierran en dos años. Por el contrario, las que miden más de $1 \mathrm{~cm}$ no cierran sino hasta alrededor de los 4 años o, en algunos casos, nunca. Por lo tanto, en términos generales, en pacientes asintomáticos, se recomienda la reparación de la hernia umbilical solo si el defecto persiste más allá de 4 a 5 años; o, bien, si el defecto es mayor de 1 a $2 \mathrm{~cm}$, está agrandando, se ha vuelto sintomático o se ha asociado con una complicación, su reparación puede considerarse antes de los 4 años. ${ }^{3}$

Las complicaciones de una hernia umbilical que requieren intervención quirúrgica inmediata son: encarcelamiento o estrangulación y, en casos extremadamente raros: ruptura, cuando la piel sobre la hernia se rompe, y expone el tejido dentro del saco herniario. Estas complicaciones son realmente excepcionales. ${ }^{4}$

La ruptura puede ser precipitada por un aumento repentino de la presión intraabdominal, como resultado del Ilanto o la tos repetida. El incremento de la presión intraabdominal durante el llanto se trasmite directamente a la piel, lo que produce isquemia y ulceración de la piel que lo recubre. La ruptura también puede ser precipitada por daño a la piel suprayacente, como consecuencia de un traumatismo o sepsis. ${ }^{5,6}$

En algunos niños la hernia umbilical se trata mediante la aplicación de medicamentos tradi- cionales que, a menudo, causan inflamación, necrosis $y$, a veces, gangrena de la piel que produce úlceras de secreción, que a su vez pueden precipitar la ruptura espontánea de la hernia. ${ }^{7}$

\section{CASO CLÍNICO}

Niña de 2 meses, ingresó al servicio de Urgencias pediátricas referida con el diagnóstico de evisceración. Antecedentes patológicos: hernia umbilical desde el nacimiento.

La madre refirió la colocación de una canica sobre el defecto umbilical, a los diez días después, al revisarla y tratar de extraerla, obtuvo las asas intestinales.

Signos vitales durante la exploración física: presión arterial 70-43 $\mathrm{mmHg}$, frecuencia cardiaca $140 \mathrm{lpm}$, frecuencia respiratoria $36 \mathrm{rpm}$, temperatura $36.5{ }^{\circ} \mathrm{C}$. Peso y talla actual 3100 $\mathrm{g}, 49 \mathrm{~cm}$, respectivamente.

Desde el punto de vista clínico la paciente se encontró hemodinámicamente estable, la piel sobre la hernia se abrió dejando al descubierto las asas intestinales (Figuras 1 y 2). Los tejidos circundantes parecían edematosos, sin evidencia de infección o necrosis. El abdomen era suave y no distendido, sin datos de irritación peritoneal. El intestino eviscerado se cubrió con una gasa estéril empapada en solución salina.

Los reportes de los exámenes de laboratorio y gasometría sin alteraciones. En las radiografías toracoabdominal antero-posterior y lateral de abdomen (Figuras 3 y 4) se observó una canica en la cavidad abdominal. Con lo anterior se estableció el diagnóstico de ruptura de saco herniario umbilical asociada con la colocación de un cuerpo extraño, con evisceración.

Se realizó intervención quirúrgica de urgencia, con plastia e introducción de las asas intestina- 
González-Rodríguez LE, et al. Ruptura de saco herniario umbilical con evisceración

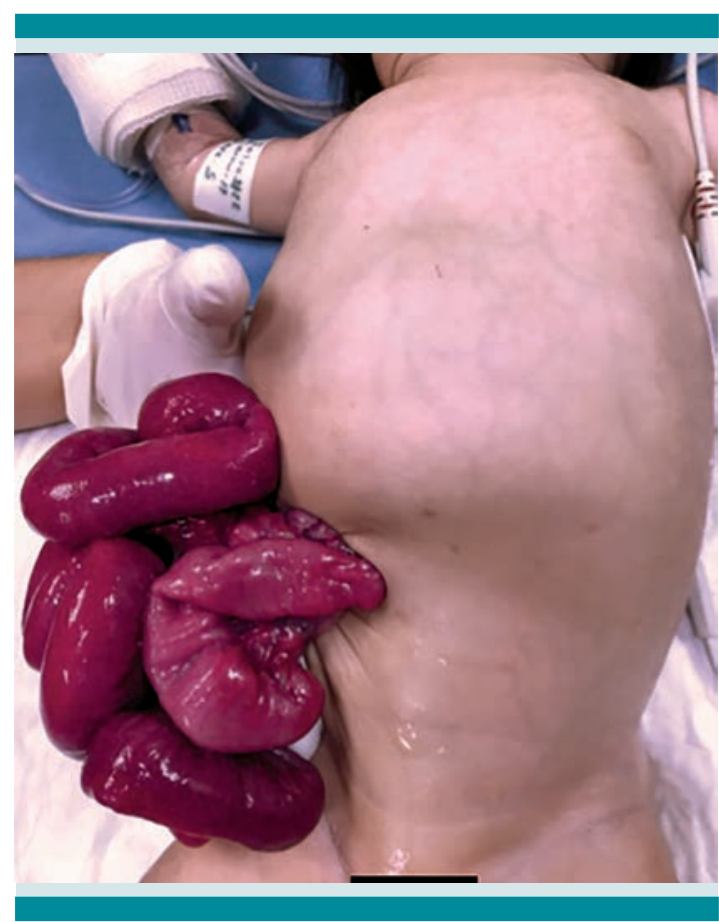

Figura 1. Hernia umbilical rota con intestino eviscerado.

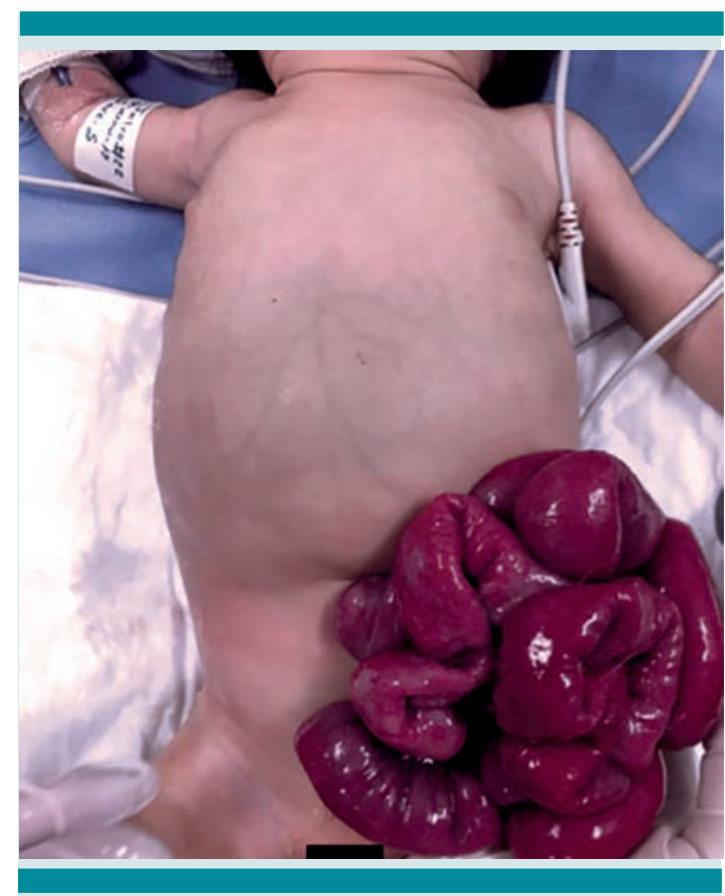

Figura 2. Hernia umbilical rota con intestino eviscerado.

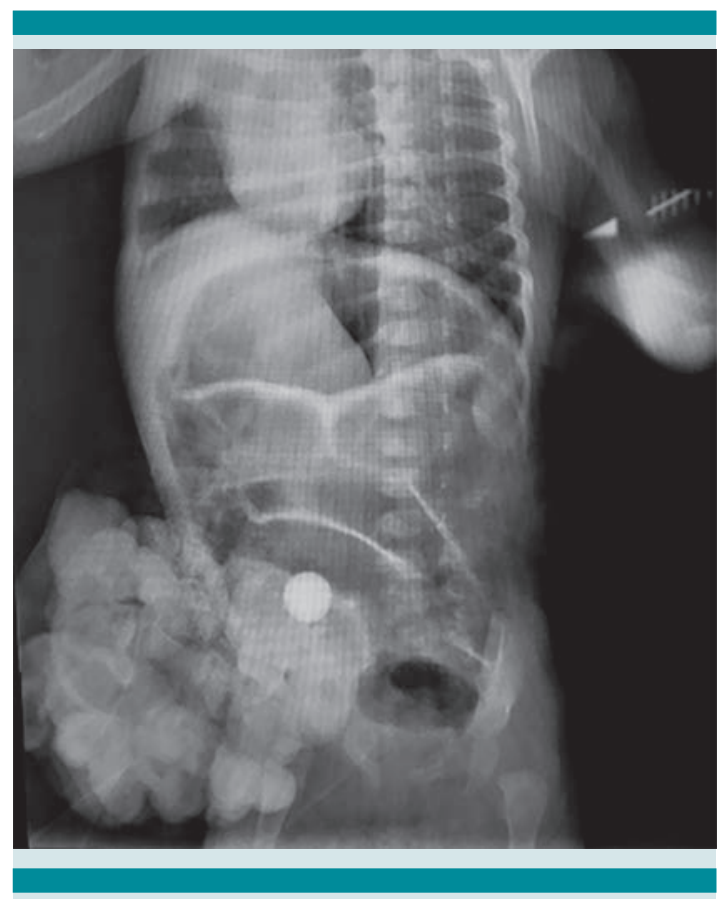

Figura 3. Radiografía toracoabdominal anteroposterior con cuerpo extraño (canica) en la cavidad abdominal.

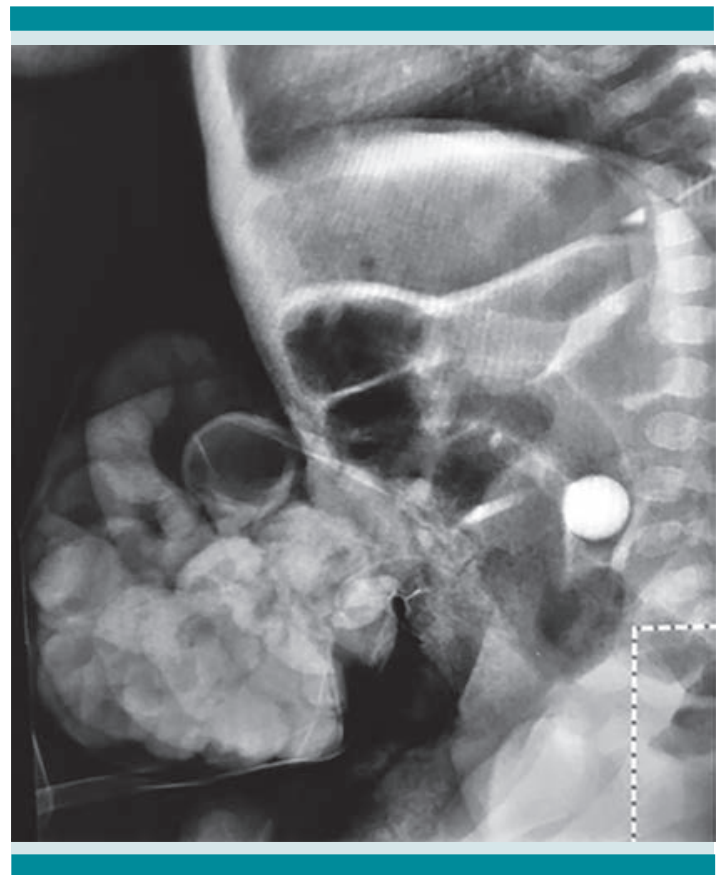

Figura 4. Radiografía lateral de abdomen con cuerpo extraño (canica) en la cavidad abdominal 
les, sin contratiempos ni complicaciones (Figura 5 y 6). Los hallazgos quirúrgicos revelaron la existencia de un objeto extraño en la cavidad (canica) y las asas del intestino delgado evisceradas. El tamaño del defecto de la pared era de $2 \mathrm{~cm}$ de diámetro, a la altura del ombligo.

El intestino se encontró sano, sin evidencia de isquemia y fue devuelto a la cavidad abdominal sin tensión. Se extirparon el saco herniario y la piel umbilical redundante. Se realizó una umbilicoplastia para lograr la restauración cosmética (Figura 7). La paciente tuvo un curso
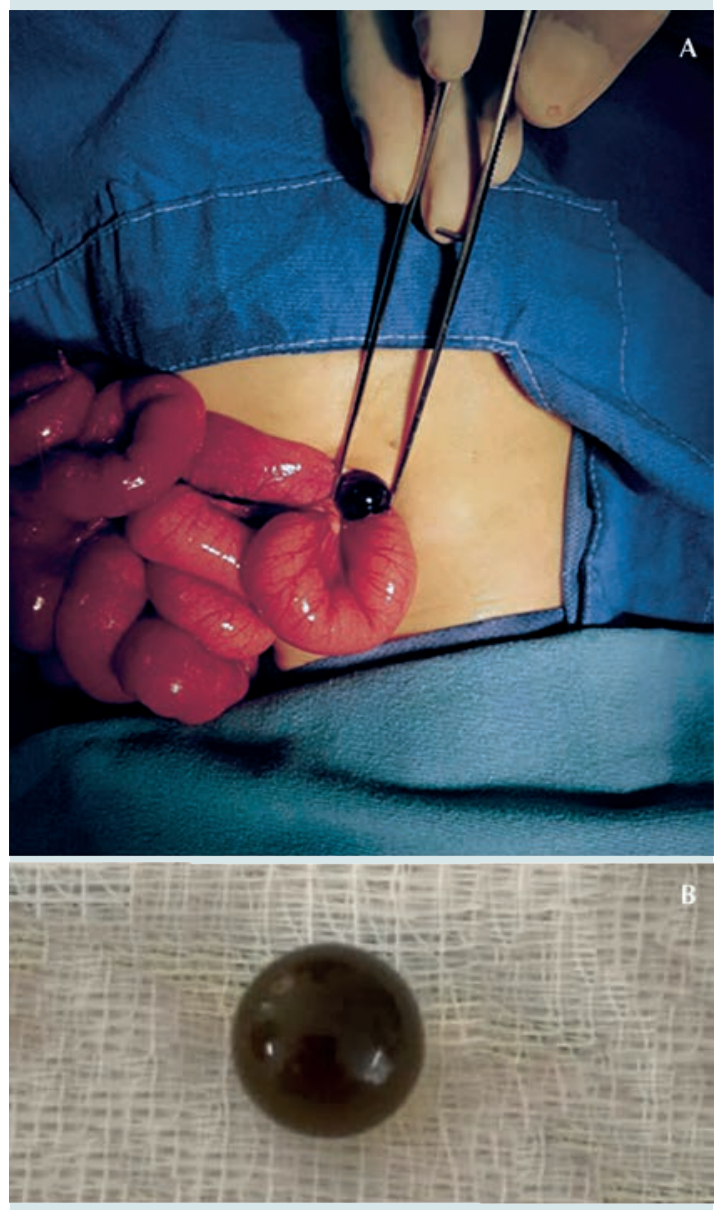

Figura 5. Extracción del cuerpo extraño (canica) de la cavidad abdominal (A). Canica extraída (B).

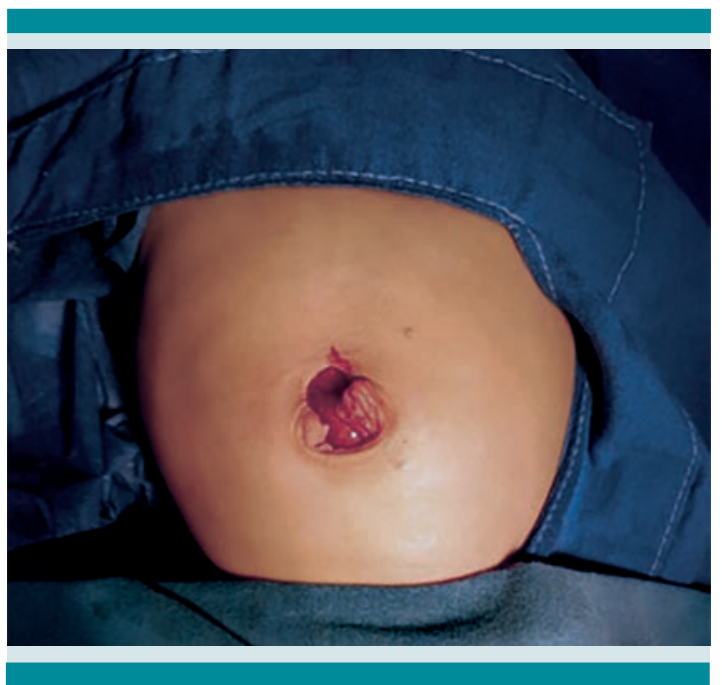

Figura 6. Imagen posterior a la introducción del intestino delgado eviscerado a la cavidad.

posoperatorio sin complicaciones. Se recuperó por completo y, posteriormente, fue dada de alta en condiciones satisfactorias para continuar el tratamiento en su domicilio.

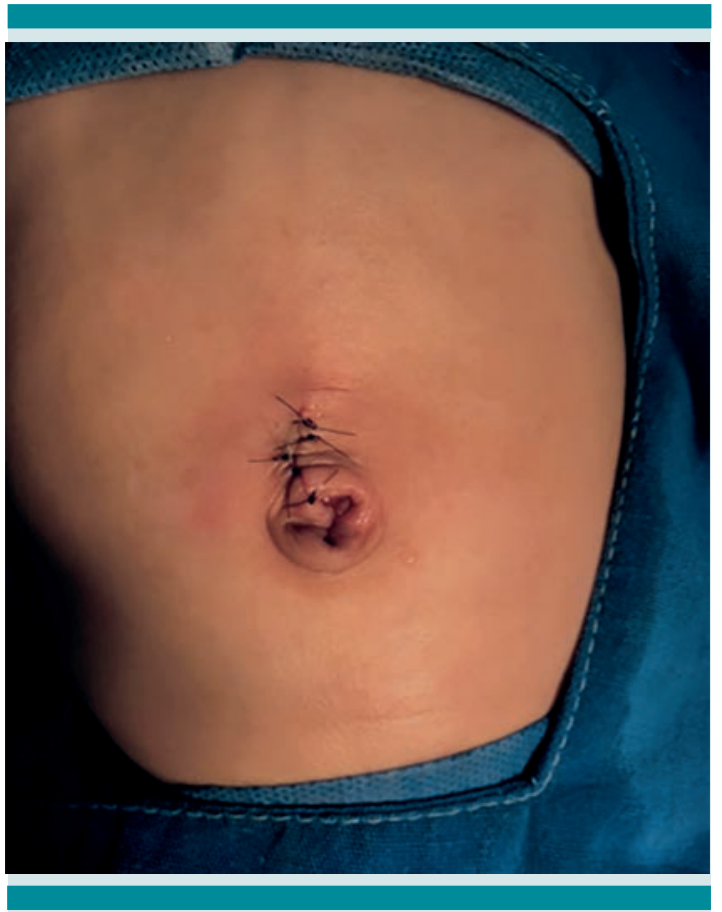

Figura 7. Resultado posterior a la umbilicoplastia. 


\section{DISCUSIÓN}

Las hernias umbilicales son más evidentes durante los episodios de llanto, esfuerzo y defecación; pueden generar una protrusión considerable del saco y su contenido. ${ }^{8}$

La ruptura espontánea de una hernia umbilical en niños es extremadamente rara y ocurre, sobre todo, en pacientes cirróticos, desnutridos o sépticos. Las hernias grandes son más propensas a la ruptura espontánea: la piel se estira y luce brillante. La tasa de mortalidad es baja: existen dos casos de muerte reportados. En ambos casos la atención fue tardía. 4,8

La ruptura espontánea de la hernia umbilical suele suceder en el trascurso de la primera infancia. En el momento de la ruptura la paciente tenía dos meses de edad y desnutrición leve. Esta condición coincide con la de otros informes en los que los pacientes son menores de cuatro meses, desnutridos o sépticos..$^{5,9}$

La ruptura espontánea de una hernia umbilical sigue siendo excepcional, hay varios factores que se cree la precipitan, incluida la edad, el tamaño del defecto, la sepsis o ulceración umbilical y cualquier condición que eleve la presión intraabdominal (llanto, tos, neumonía, ventilación con presión positiva, ascitis o patología intraabdominal). ${ }^{10}$ También puede ser el resultado del daño a la piel que recubre, ya sea en forma de ulceración o sepsis umbilical, la que se ha notificado en $38 \%$ de los casos de evisceración espontánea, o debido a la falla en la cicatrización de una herida umbilical reciente, o una cicatriz débil que no puede soportar el estrés de la presión intraabdominal elevada asociada con la tos, los vómitos o la defecación en los primeros años de vida. ${ }^{5,10}$ Los factores desencadenantes de la ruptura también pueden incluir traumatismo local., 2,5 La colocación de cuerpos extraños (canicas, monedas, entre otros) es un factor de riesgo de sepsis umbilical que precipita el encarcelamiento $y$, por lo tanto, daña la piel sobre la hernia y expone el tejido dentro del saco herniario. ${ }^{10}$

En el caso aquí reportado fue determinante la colocación de una canica sobre la hernia umbilical como método para su reducción, objeto comunmente utilizado en algunos sectores de nuestra población, que condicionó que la piel sobre la hernia se adelgazara y ulcerara por necrosis por presión. El intertrigo en el pliegue inferior entre la hernia y la pared abdominal es común debido a una combinación de sudor, calor y fricción. Esto, a su vez, dañó la piel suprayacente. Lo anterior, y ante el incremento de la presión intraabdominal por llanto, tos u otro factor, precipitó la ruptura espontánea del saco herniario. Todos estos factores contribuyeron al encarcelamiento y eventual evisceración.

Ante la ruptura de una hernia umbilical, el tratamiento es la prevención de la pérdida de calor de la cavidad abdominal abierta y el intestino expuesto al envolver el intestino herniado en una gasa empapada en solución salina caliente, el secado del niño y el uso de un calentador de calor radiante. El niño debe colocarse en el lado derecho para evitar el acodamiento del mesenterio y la isquemia intestinal resultante. ${ }^{4}$

\section{CONCLUSIÓN}

El curso sin complicaciones y el alivio espontáneo de la mayor parte de las hernias umbilicales pediátricas es un fenómeno bien reconocido. De hecho, esta es la única hernia de la pared abdominal que puede tratarse sin intervención quirúrgica. En comparación con los pacientes adultos con hernia umbilical, en particular las mujeres embarazadas o pacientes cirróticos con ascitis, los niños rara vez experimentan complicaciones, como el encarcelamiento o el estrangulamiento. Los informes de evisceración son aún más excepcionales. ${ }^{11}$ La ruptura espon- 
tánea con evisceración es extremadamente rara, en más de 50 décadas hay 19 casos reportados de ruptura espontánea de hernia umbilical en la bibliografía. El que aquí se reporta es el primero en la bibliografía mexicana.

\section{REFERENCIAS}

1. Lissauer T, Clayden G. Illustrated Textbook of Paediatrics. $3^{\text {rd }}$ ed. London: Mosby, 2007.

2. Armstrong 0 . Hernies ombilicales. Rev Prat. 2003;53(15):1671-6.

3. Zendejas B, et al. Fifty-three-year experience with pediatric umbilical hernia repairs. J Pediatr Surg. 2011;46(11):2151 https://doi.org/10.1016/j.jpedsurg.2011.06.014

4. Gupta R, et al. Spontaneous rupture of a congenital umbilical hernia in an infant: A rare complication. Bangladesh J Medical Science 2015;14(1). https://doi.org/10.3329/ bjms.v14i1.21574

5. Ahmed A, et al. Spontaneous rupture of infantile umbilical hernia: report of three cases. Ann Trop Paediatr. 1998
Sep;18(3):239-41. https://doi.org/10.1080/02724936.1 998.11747953

6. Kittur DH, et al Rupture of umbilical hernia with evisceration in a newborn. A case report. J Neonatal Surg. 2017 Aug 10;6(3):67. doi: 10.21699/jns.v6i3.565

7. Ginsburg BY, et al. Spontaneous rupture of an umbilical hernia with evisceration. J Emerg Med. 2006;30(2):155-57. https://doi.org/10.1016/j.jemermed.2005.05.017

8. Thomson $\mathrm{WL}$, et al. A literature review of spontaneous evisceration in paediatric umbilical hernias. Pediatr Surg Int. 2012 May;28(5):467-70. https://doi.org/10.1007/ s00383-012-3076-8

9. Bain IM, et al. Spontaneous rupture of an infantile umbilical hernia. Br J Surg 1995; 82(1):34-35. https://doi. org/10.1002/bjs.1800820114

10. Chouikh T, et al Uncommon Complication of Paediatric Umbilical Hernia: Spontaneous Evisceration. Case Report and Literature Review. J Pediatr Neonatal Care. 2016;5(6): 00202.

11. Weik J, et al. An unusual case of umbilical hernia rupture with evisceration. Journal of Pediatric Surgery. 2005;40(4):E33-E35. https://doi.org/10.1016/j.jpedsurg.2005.01.026 